\section{Cahiers de Narratologie}

Analyse et théorie narratives

17 | 2009

Stéréotype et narration littéraire

\title{
Avant-propos : stéréotype et narration littéraire
}

\section{Antonello Perli}

\section{OpenEdition}

Journals

Édition électronique

URL : http://journals.openedition.org/narratologie/1338

DOI : 10.4000/narratologie.1338

ISSN : 1765-307X

Éditeur

LIRCES

\section{Référence électronique}

Antonello Perli, « Avant-propos : stéréotype et narration littéraire », Cahiers de Narratologie [En ligne], 17 | 2009, mis en ligne le 22 décembre 2009, consulté le 30 avril 2019. URL : http:// journals.openedition.org/narratologie/1338; DOI : 10.4000/narratologie.1338

Ce document a été généré automatiquement le 30 avril 2019

\section{(c) (i) $\odot$}

Cahiers de Narratologie - Analyse et théorie narratives est mis à disposition selon les termes de la licence Creative Commons Attribution - Pas d'Utilisation Commerciale - Pas de Modification 4.0

International. 


\title{
Avant-propos : stéréotype et narration littéraire
}

\author{
Antonello Perli
}

1 Consacré aux rapports que le stéréotype entretient avec la narration littéraire, ce numéro des Cahiers de narratologie présente des études qui, fondées sur une considération aussi bien poétologique que sociologique de la notion qui est au cœur de la réflexion, prennent comme point de mire la nature et les ressources du stéréotype en tant que phénomène textuel, son environnement conceptuel et son soubassement épistémologique, ses formes et ses finalités d'inscription dans le récit, ses effets esthétiques et ses implications axiologiques.

2 S'attachant à identifier des ressorts ou des mécanismes textuels relevant de la stéréotypie comme phénomène général de l'œuvre littéraire, à étudier la manière dont le texte se construit sur des constituants stéréotypiques (formels ou idéologiques) ou renvoie intertextuellement ou architextuellement au champ de la stéréotypie, les articles ici réunis saisissent les diverses facettes de cette notion, la mettent à l'épreuve en explorant sa plurivocité fonctionnelle et sémantique.

3 Couvrant un large spectre en termes de périodes et de courants littéraires, d'univers linguistiques et socio-culturels (du Siglo de Oro au XIX ${ }^{\mathrm{e}}$ siècle français, des avant-gardes des années vingt au postmodernisme américain et à l'actualité romanesque espagnole ou d'Afrique francophone), ces articles fournissent les éléments d'une typologie (repérage des formes revêtues par le stéréotype), d'une topographie (description de ces formes, de leurs contours et de leurs modalités d'insertion dans le tissu diégétique, tant au plan thématique qu'au niveau discursif) et d'une herméneutique du stéréotype (décryptage des enjeux éthiques, idéologiques et poétiques).

4 S'appuyant sur un large éventail d'ouvrages théoriques qui nourrissent la réflexion produite par leurs auteurs et qui enrichissent l'appareil des références bibliographiques, ces articles mènent de concert le questionnement théorique, la problématisation critique et l'étude d'œuvres singulières qui relèvent bien davantage de la littérature dite «blanche » (dont est constitué l'essentiel du corpus représenté) que de la littérature " de genre » (à laquelle sont consacrés les deuxième et troisième articles) ou de la « littérature 
à stéréotypes ${ }^{1}$ ", l'un des objectifs des analyses étant d'ailleurs l'approche du phénomène d'identité et de "frottement " génériques des œuvres (en fonction des degrés divers d'assimilation ou de subversion, d'intégration ou de rejet du topique), ainsi que l'approche du problème théorique touchant la relation entre généricité et stéréotypie, problème qui constitue l'objet direct et l'enjeu fondamental de la réflexion livrée par Raphaël Baroni dans l'article liminaire de ce numéro. S'attachant à cerner le phénomène de la généricité et s'employant à redéfinir son champ d'application, Baroni prend en effet comme point de mire le problème de l'extension du concept de généricité et de sa définition par rapport à la question plus générale de la stéréotypie. Sensible aux manifestations d'une rupture de l'horizon d'attente générique, Baroni démontre - en exemplifiant son propos par l'analyse d'un fragment d'une nouvelle de Marcel Aymé qu'un détournement de la régularité attendue, bien que ressortissant à une relation du texte à un architexte, c'est-à-dire à une régularité instituée au sein de l'interdiscours, relève moins de la généricité que de la stéréotypie. Et l'auteur de mettre l'accent sur la valeur opérationnelle du concept de stéréotype : «Pour autant qu'on débarrasse le terme 'stéréotype' de sa connotation péjorative, il permet de définir un concept d'une très grande extension et d'une grande souplesse d'usage, particulièrement pratique pour analyser les questions liées aux régularités instituées au sein de l'interdiscours, dans toutes les formes que ces dernières peuvent prendre. Dans ce contexte, le genre apparait alors comme une forme particulière de stéréotypie: c'est un stéréotype générique ». Convoquant les théories qui définissent le genre par rapport aux notions de " préjugé " ou d'« horizon d'attente » et mettant en évidence plus particulièrement les limites de celles qui le rattachent à la catégorie du "scénario ", Baroni affirme que l'on ne peut confiner le concept de généricité dans le cadre des régularités attendues: «Lorsqu'on aborde la question plus générale des régularités instituées au sein de l'interdiscours [...] peut-être serait-il préférable de s'en tenir aux termes plus généraux de 'stéréotype' (Dufays), d' 'horizon d'attente' (Jauss), d' 'architexte' (Genette) ou de 'préjugé' (Gadamer), afin d'éviter de confondre le phénomène global des généralités intermédiaires qui nous permettent de vivre en société avec la notion plus étroite - mais aussi plus précise et plus efficace pour interpréter certains effets littéraires - de genre ». Au-delà du débat théorique captivant auquel il nous convie, l'intérêt de cet article de Raphaël Baroni tient aussi aux implications herméneutiques de la réflexion et des analyses qu'y livre son auteur, pour qui il s'agit de montrer que la rupture d'un horizon d'attente «n'est pas simplement l'affirmation de la singularité du texte, mais bien un effet de sens qui ne peut être actualisé que dans la mesure ou l'on établit un rapport entre le texte et une régularité attendue du discours. [...] Par ailleurs, Gadamer insistait sur le fait que c'était par la 'résistance' que le texte oppose à nos présuppositions (ou à nos 'préjugés') que ce dernier pouvait faire valoir son contenu de vérité. Cela signifie que les savoirs que nous nous constituons progressivement à travers nos pratiques socio-discursives antérieures, bien qu'indispensables à notre compréhension des textes, doivent malgré tout nécessairement être dépassés dans le processus interprétatif pour remplir leur rôle jusqu'au bout ; l'interprétation ne peut se résumer à une simple réactualisation circulaire des codes mais tend toujours à produire un sens et un savoir inédits ».

5 Envisageant la stéréotypie sous les espèces d'un discours critique, d'une catégorie ou d'une figure narrative, d'une structure référentielle, d'une modalité énonciative, l'examinant dans ses dispositifs de suturation sur le récit, dans ses traits de métissage discursif, dans ses interférences séquentielles, dans sa portée idéologique aussi, les auteurs des articles de ce numéro mènent l'enquête dans les différents champs de la 
textualité: l'intratextualité, sur laquelle se fondent les analyses de la stylistique discursive, de la structuration de l'illusion référentielle, des stratégies de fictionnalisation (Le Blanc, Fournié-Chaboche, Marion François), mais à partir de laquelle se produit aussi un embrayage résolu vers l'extratextualité (Tandia Mouafou, Di Benedetto); l'intertextualité des pratiques autostéréotypiques (Delaune), parodiques (Le Blanc, Cécile François) ou hypertextuelles (Rialland); la métatextualité du discours autoréflexif et de l'écriture métafictionnelle (Karoui-Elounelli) ; l'architextualité (Anissa Belhadjin montrant comment les romans noirs s'inscrivent dans le vaste ensemble que constitue le " genre noir » et jouent sur ses codes, Marion François relevant, à propos du genre policier, la mise en question des pratiques taxinomiques, les affiliations « muettes " ou favorisées par le paratexte, le dialogisme et la reprise topique des thèmes dans un jeu de métissage).

Dans le cadre d'une interaction analytique entre plusieurs aires de la textualité, Alissa Le Blanc montre comment Laforgue s'emploie à défaire les mécanismes de l'illusion référentielle, notamment grâce aux interventions intempestives du narrateur. L'auteur des Moralités légendaires souligne à outrance les emprunts à des œuvres connues ou la reprise de topoi littéraires. Les nouvelles étoilent vers d'autres textes dans une danse étourdissante de références ayant pour conséquence le parasitage des effets de réel et l'insistance sur la dimension métatextuelle de l'œuvre, qui multiplie les allusions au processus de l'écriture et à la fiction. La parodie, diffuse, voit sa cible se diluer et permet de redéfinir le cadre du genre de la nouvelle conventionnelle. Cécile François met en relief la double relation intertextuelle et métatextuelle que la trilogie de Jardiel Poncela entretient avec la littérature sentimentale de masse, les pratiques démystificatrices et parodiques de l'auteur dans la récupération des invariants du roman sentimental, et elle met l'accent sur la récurrence des parabases et sur la charge ironique, voire sarcastique, des commentaires de l'auteur à l'encontre de ce genre de littérature. La double marque de la métatextualité et de l'intertextualité est également repérée par Salwa KarouiElounelli dans son analyse de la portée métafictionnelle, autoréflexive des récits de John Barth : celle-ci se fonde sur la spécularité intratextuelle (le personnage est le double de l'auteur) et sur la pratique hypertextuelle (les réflexions sur la création littéraire procèdent d'un besoin d'imiter ou de se "greffer » sur les textes classiques), le mode parodique des jeux intertextuels s'articulant architextuellement à une refonte du récit de la quête et du roman de voyage.

7 Adoptant tour à tour ou conjointement la perspective de l'auteur et celle du lecteur, les contributions réunies dans ce numéro sont orientées selon différentes perspectives: esthétique (stéréotype fonctionnel à visée scripturale, enjeu de l'écriture); rhématique (niveau discursif, stratégie énonciative) ; pragmatique (relation auteur/lecteur, effet sur le narrataire/lecteur); épistémologique (champ conceptuel de la stéréotypie); générique (poétique des genres littéraires); sociocritique (contexte, idéologie, référentialité). Sur la base de ces diverses perspectives (parfois exclusives ou privilégiées, parfois coexistant au sein d'une lecture multifocale), ces études abordent les domaines de recherche d'une sémantique du stéréotype, d'une poétique et d'une rhétorique du stéréotype, d'une éthique du stéréotype.

8 Dans le cadre d'une sémantique du stéréotype, Alissa Le Blanc tente dans la première partie de son article de définir la notion de "poncif » par rapport aux termes voisins de "stéréotype », de « lieu commun", de «topos », de «topique » ou de «cliché », pour en dégager les spécificités critiques et conceptuelles, apportant ainsi un éclairage sur la 
notion de poncif, dont il s'agit de mettre en évidence à la fois les ambiguïtés sémantiques et la pertinence critique. Constatant que le vocable stéréotype est souvent assimilé à des termes qui lui sont voisins (cliché, poncif, lieu commun ou encore idée reçue), Vincent Stohler propose une terminologie qui se fonde sur les traits définitoires retenus par les dernières études sur le sujet.

Mettant en lumière la visée axiologique ou l'effet idéologique du stéréotype envisagé comme véhicule d'une vision du monde, de l'homme, de la société, certaines analyses se déploient dans le domaine d'une éthique du stéréotype. Dans son article consacré aux derniers ouvrages du romancier camerounais Mongo Beti, J.-J.R. Tandia Mouafou étudie l'utilisation romanesque du stéréotype en l'agençant à la problématique d'une construction textuelle des valeurs, ce qui, dans une perspective sociocritique, le conduit à voir dans ces romans l'expression d'une métaphore des sociétés africaines postcoloniales. Dans Nosotras que no somos como las demás, le roman de Lucía Etxebarria analysé par Christine Di Benedetto, se côtoient deux groupes de femmes caractérisés par deux stéréotypes dont les formes et les modes d'insertion dans le texte sont différents : charge est laissée au récepteur de procéder à la reconstruction des deux stéréotypes en récupérant les schèmes, explicites ou implicites, éparpillés dans le texte. La confrontation de ces deux stéréotypes a une visée idéologique de positionnement d'un groupe de femmes «nouvelles» par rapport à l'autre groupe plus vaste, plus ancien et mieux implanté dans la société espagnole actuelle. Il en résulte un jugement de valeur en faveur $\mathrm{du}$ premier et une affirmation axiologique quant à l'espace social que chacun occupe. Dans son étude des proverbes dans le Quichotte, Sonia Fournet-Pérot s'attache à décrire la fonction de communication entre les différents émetteurs et récepteurs de ce stéréotype culturel et son articulation à la stratégie énonciative et au message axiologique de l'œuvre cervantine, qui mêle ironie, humour et absurde pour présenter une vision critique de la société de l'époque. Mathias Ledroit analyse le stéréotype littéraire du bandoulier catalan dans des œuvres de Cervantès, Lope de Vega et Tirso de Molina, et met en parallèle ce personnage avec la description du stéréotype social figurant dans les ouvrages juridiques, historiques et géographiques du $\mathrm{XVII}^{\mathrm{e}}$ siècle qui s'interrogent sur les origines du phénomène.

10 Une autre série d'articles s'inscrit conjointement dans les domaines d'une poétique $d u$ stéréotype et d'une rhétorique du stéréotype. Après avoir repéré et identifié dans le corpus étudié les différentes formes du stéréotype, et analysé leurs modalités d'insertion dans le texte, Anissa Belhadjin montre que les stéréotypes narratifs ont une fonction pragmatique et constituent un « code » qui programme la réception du roman noir, mais qu'ils peuvent aussi déjouer les attentes du lecteur et contribuer à modifier le genre noir et à marquer son évolution. Pour Marion François le contrat implicite du genre policier donne des garanties au lecteur, qui sait qu'il va trouver dans ce roman une structure et des thèmes connus et pouvoir jouir du jeu éventuellement instauré avec ce champ ou être déjoué dans ses prévisions par toutes sortes de perversions du contrat. L'auteur novateur peut ainsi amener le lecteur à accepter des modifications importantes, allant jusqu'à une fin ouverte ou déceptive. Le cliché provoque l'écriture, il en dit les impasses, délie la langue de l'écrivain en lui fournissant l'équivalent d'une portée musicale où seraient inscrites quelques notes, et/ou en le provoquant à dynamiter les scénarios tout faits. D'où l'essaimage du genre dans la littérature dite «blanche ». L'auteur capture le lecteur dans les filets des stéréotypes, lui fournissant une lecture fantasmatique qui l'empêche de voir les indices, souvent eux-mêmes clichés. Instaurant une complicité entre auteur et lecteur 
dans le bonheur du déjà-vu et le jeu subversif avec le modèle, le cliché permet au lecteur, en lui donnant une assise, d'appréhender des œuvres plus expérimentales ou plus complexes. Par ailleurs, Marion François considère que l'étude du stéréotype dans ce genre littéraire permet d'interroger les conséquences du classement et du jugement génériques qui font du roman policier et du cliché deux mal-aimés de la littérature. Marta Cuenca-Godbert étudie le retour spéculaire du personnage de don Quichotte sur lui-même en tant que personnage littéraire dans la deuxième partie cervantine (1615) en regard avec la première (1605) et la deuxième partie apocryphe (1614). La reconnaissance du personnage littéraire par d'autres à l'intérieur de la fiction est marquée d'un refus de la part de don Quichotte de devenir le stéréotype fabriqué dans la suite d'Avellaneda. Ce n'est pas la mort du personnage à la fin de l'œuvre qui empêche la production de suites sur le modèle chevaleresque, mais bien la considération de don Quichotte comme personnage mythique et non comme stéréotype. Sylvie Fournié-Chaboche étudie les stéréotypes lexicalisés dans le roman de Javier Tomeo Diálogo en Re Mayor et montre que ces formes fixes, parce qu'elles sont porteuses de répétitions connotées, contribuent à une « esthétique de la répétition ». Loin de la trivialité supposée de ces expressions figées imagées, la présence de celles-ci en tant que répétitions connotées constitue un véritable enjeu d'écriture: le principe de répétition innerve en effet tout ce roman construit comme une fugue musicale. Salwa Karoui-Elounelli s'attache à repérer dans la fiction de John Barth les pratiques narratives de la répétition ironique et subversive des formes et motifs romanesques classiques, et notamment la remise en question du récit de la quête et du roman de voyage dans le roman postmoderne américain. Elle démontre que la répétition ironique des textes littéraires archétypaux maintient le lecteur dans l'ambiguïté d'une complicité avec le texte parodiant, doublée d'une distance critique à l'égard des jeux intertextuels démystifiés. Les risques de stéréotypage activés par la répétition ironique des motifs et des figures des textes canoniques, offrent au récit parodique postmoderne un espace d'autoréflexivité littéraire où se développe une esthétique du dédoublement qui se caractérise par sa visée métacritique. Dans la seconde partie de l'article d'Alissa Le Blanc, l'analyse des stratégies d'écriture dans les Moralités légendaires de Jules Laforgue tend à montrer que l'écrivain utilise les conventions narratives pour mieux les détourner et les subvertir, afin d'en critiquer le caractère éculé. Cette déconstruction des mythes ou du romanesque traditionnel aboutit surtout à la recomposition d'une forme, fondée sur la parodie, qui contribue à redéfinir le genre de la nouvelle. Ces détournements des codes et des structures narratives conventionnels déjouent les habitudes de réception du lecteur et cherchent à développer de nouvelles formes de complicité, fondées sur le réinvestissement ludique d'une mémoire culturelle commune mais aussi sur le dénudement des procédés fictionnels. Le narrataire a ainsi l'impression d'être plus étroitement associé à la construction du texte et d'entrer dans les coulisses de sa composition. Alissa Le Blanc montre ainsi comment Laforgue tente d'instaurer de nouveaux modes d'échange avec son lecteur, qui doit accepter d'être déçu, dépisté et sollicité à chaque instant. L'écrivain joue à la fois de la complicité culturelle qu'il partage avec son destinataire et de la défamiliarisation qu'il lui fait subir en subvertissant les codes fictionnels et en détournant des références bien connues. On touche là aux enjeux cruciaux de l'écriture des Moralités, qui cherchent à transformer le poncif narratif en le déconstruisant pour en proposer des reconfigurations inédites, où l'humour a toute sa place. La stratégie scripturale de Laforgue est à la fois habile et féconde : il s'agit de faire du neuf avec de l'ancien plutôt que de prétendre faire table rase des clichés. En ce sens, le but n'est pas seulement ludique: la démarche répond à 
l'intention d'écrire en échappant au banal, tout en sachant que, précisément, il est impossible d'éviter les lieux communs. Vincent Stohler étudie la manière dont Flaubert récupère les stéréotypes présents dans les physiologies des encyclopédies de mœurs du début du XIX siècle et définit leurs modalités d'insertion dans Bouvard et Pécuchet en montrant que les stéréotypes influent directement sur la composition des personnages (traits physiques, caractériels, axiologiques), mais aussi sur la structure du texte. Stohler attire ainsi l'attention sur la subtilité du travail de stéréotypie de Flaubert, qui permet à l'écrivain de dénoncer de manière originale la présence de schèmes figés dans les discours scientifiques de son époque. Analysant l'utilisation du stéréotype romanesque dans les romans surréalistes des années vingt, Ivanne Rialland montre comment il contribue à l'agencement de l'intrigue et à la réalisation d'un romanesque opposée à l'attitude réaliste dénoncée par André Breton. L'usage du stéréotype romanesque par le surréalisme n'est pas seulement critique ou ludique: il correspond à une esthétique romanesque articulée à une éthique. Les ressorts romanesques, même les plus éculés, contribuent en effet à repassionner le rapport à un réel dont le réalisme ne sait faire voir que l'usure. Cécile François s'intéresse à la récupération ludique des procédés d'écriture du roman d'amour dans la trilogie d'un écrivain espagnol, Jardiel Poncela, qui s'applique à dénuder les paradigmes narratifs conventionnels pour mieux les subvertir. Elle analyse la mise en place d'une relation de complicité entre l'auteur et le lecteur, qui est invité à participer activement à l'entreprise de mise à distance et de démontage des stéréotypes et des clichés de la littérature sentimentale. L'étude de Cécile François montre ainsi que la subversion des stéréotypes de la littérature sentimentale n'est pas une fin en soi mais qu'elle débouche sur la mise en place de nouvelles pratiques d'écriture liées à l'esthétique des avant-gardes. La dénonciation systématique du stéréotype vise également à bloquer le processus d'identification du lecteur pour l'inciter à adopter une distance critique par rapport au texte, et pour le faire passer ainsi du rôle de simple consommateur à celui de sujet pleinement conscient des enjeux de la lecture.

\section{NOTES}

1. Voir à ce sujet « Littérature à stéréotypes ", sous la direction de Odile Gannier, Loxias, n. 17 (14 juin 2007), http://revel.unice.fr/loxias/document.html?id=1741.

\section{AUTEUR}

\section{ANTONELLO PERLI}

Maître de conférences HDR à l'Université de Nice-Sophia Antipolis, CIRCPLES 